\title{
A Novel Management Methodology or Ad-Hoc Wireless Networks to Improve the Routing Efficiency with Adaptive Broadcasting Range under Lower Transmitting Energy
}

\author{
Yung-Fa Huang ${ }^{1}$, Hsing-Wen Wang ${ }^{2}$ and Hui-Chao Hsu ${ }^{1}$ \\ ${ }^{1}$ Department of Information and Communication Engineering, \\ Chaoyang University of Technology \\ ${ }^{2}$ Department of Business Administration, \\ National Changhua University of Education \\ Email: shinwen@cc.ncue.edu.tw
}

\begin{abstract}
By investigating the effects of nodes distribution and broadcasting range of the route request (RREQ) control packets on the routing path establishment, in this paper, we explore the energy efficiency of AODV (Ad-hoc on-demand distance vector) routing algorithms in Ad-Hoc networks. Simulation results show that with the range adaptation to the number of nodes the highest successful rate of routing path establishment is obtained. With the adaptive broadcasting range the routing efficiency can be improved. Thus, the demand transmitting energy of RREQ control packets to establish a routing path can be reduced.
\end{abstract}

Keywords- Ad-Hoc networks; AODV; Transmission rang; RREQ Control packet; Transmission power.

\section{INTRODUCTION}

The wireless network architecture can be divided into two architectures of with infrastructure and without infrastructure (Wireless Ad Hoc Networks) [1]. Wireless ad hoc network is a high mobility, dynamic topology network architecture. Therefore, the routing path can not be stably established and the route request (RREQ) packets can not be efficiently broadcasted over Ad-hoc network.

Ad-hoc network routing protocols can be broadly divided into two categories. One category is a table-driven, and the other is the source-initiated on-demand driven. In table-driven, each node periodically sends or exchange routing information to update routing tables. The nodes find a transmission path from the routing table to transmit data, such as DSDV [1]. In source-initiated on-demand driven protocols, when the node needs to send data packets to the destination, it begin to find a path to perform data transmission. The source node broadcasts RREQ control packets to the neighboring node until the destination node receives an RREQ message. The destination node send back an RREP packet to the source node. After the source node receive the RREP, it finds the available path in RREP. Then, it stops broadcasting RREQ. The AODV[2]、 DSR[3] are the examples of the on-demand driven.

In table-driven each node must be periodically exchange routing information over networks. The network resource can be consumed largely [4]. On the other hand, the on-demand driven oriented, do not need to regularly update the routing information. However, due to the dynamic changes of the Adhoc network, the links between the nodes is not permanent. To determine the correct information on the transmission path, the node would continue to broadcast the RREQ packets to perform the establishment of the path. Thus, when each node broadcasts control packets [5] largely, the node energy consumption will increase [6]. Therefore, in a constantly changing network topology structures, the path establishment is a challenging task for the power aware wireless networks [7] [8].

In this paper, we investigate the energy efficiency of routing path establishment of AODV. In Section 2, the related literature is investigated for the Ad-Hoc network routing protocols. In Section 3, the ad-hoc network models are established for simulation environments. In Section 4, the simulation results of success rate for path establishments is performed. In Section 5, some conclusions are given.

\section{AODV ROUTING ALGORITHM}

The AODV is a typically on-demand routing protocol. When the source node need to send data to destination node, and without path information of the destination node in the routing table, it would start to establish the path search process. This eliminates the need for periodic maintenance cost of the path required.

When a source node needs a path to the destination node, it broadcasts an RREQ packet to the networks, and then wait for the route reply (RREP) packet. The RREQ packet contains the source node and destination node IP address, broadcast ID, the source node and destination node sequence number, as shown in Table 1. When a node receives an RREQ packet, it records the source node IP address and broadcast ID. The source node set a preset time for the RREQ packet broadcasting. If the nodes receive the same source the IP address of the node and broadcast ID of RREQ packets in this period of time, it will discard this RREQ packet.

If this RREQ packet is not received by the destination node, the node can not determine whether the path to the destination 
exists or not. Therefore, when the destination node receives the RREQ packet, it need to send back RREP to source node. When the node receives a RREQ packet, it will establish a reverse path information to the source node in the routing table. In Fig. 1, solid line arrows represent the path of the RREQ packets, broadcasting from the source node to the destination node. All the intermediate nodes receive the RREQ packets and then broadcast this RREQ packet out. Finally, if the path exists, the destination node $\mathrm{G}$ will receive the RREQ packet.

The RREP packet will be replied to the source node A through the path. When the intermediate node receives the RREP packet, it will establish a forward path information from source node to the destination node to the routing table, and then passes to the upstream node, until the RREP packet transmitted source node A.. In Fig. 2, the dashed line arrows represent the path of the RREP packets. After the destination node $G$ receiving the RREQ packet, along the path of the RREQ packet the RREP packet is sent return to the source node A. If the source node A did not receive RREP packet in countdown timer to 0 , source node $A$ repeats the broadcast of RREQ packet. Then the number of retransmission adds one. When the number of retransmissions of source node A exceeds a preset maximum value of this entry, this connection is failed.

TABLE I. RREQ packet format

\begin{tabular}{|c|c|c|}
\hline Type & Reserved & Hop Count \\
\hline \multicolumn{3}{|c|}{ Broadcast ID } \\
\hline \multicolumn{3}{|c|}{ Destination IP Address } \\
\hline \multicolumn{3}{|c|}{ Destination Sequence Number } \\
\hline \multicolumn{3}{|c|}{ Source IP Address } \\
\hline \multicolumn{3}{|c|}{ Source Sequence Number } \\
\hline
\end{tabular}

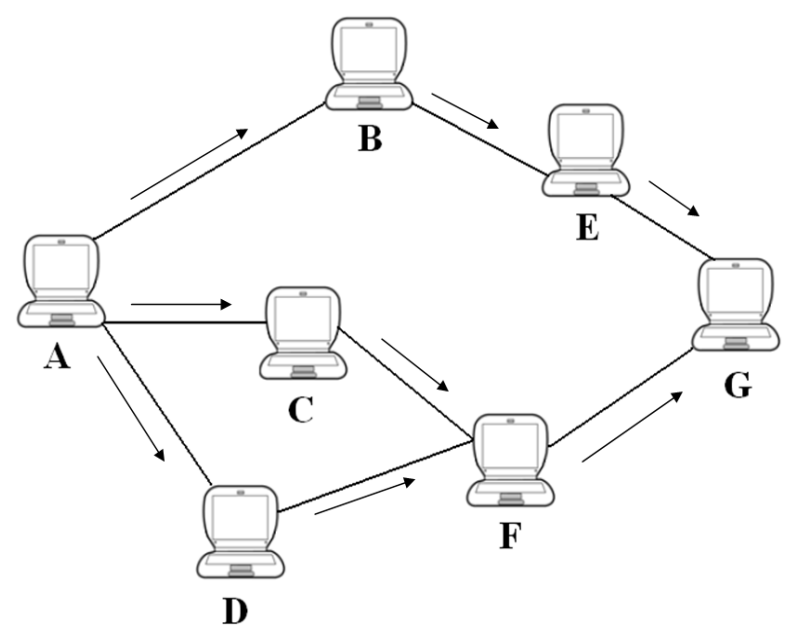

Figure 1. Source node A broadcast RREQ packet

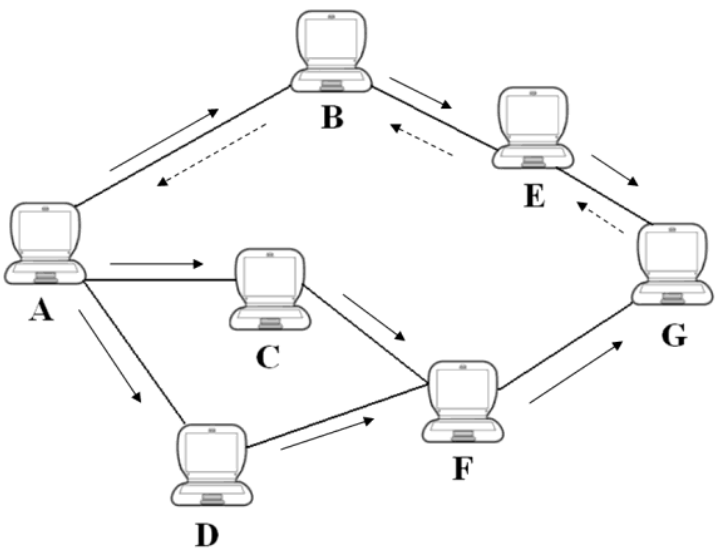

Figure 2. Destination node G return RREP packet (with dashed line)

\section{NETWORK MODELS}

In this paper, the RREQ broadcast procedure is performed by Non-Line-of-Sight(NLOS) transmission channel models [8]. In addition to consider the transmission path loss by the distance, the ground reflection model is adapted by

$$
P_{r}=\frac{P_{t} \cdot G_{t} \cdot G_{r} \cdot h_{t}^{2} \cdot h_{r}^{2}}{d^{4}},
$$

where $h_{t}$ is the transmit antenna height, $h_{r}$ is receiving antenna height, $P_{t}$ is transmit power, $G_{t}$ is the transmit antenna gain, $G_{r}$ is the receiving antenna gain, $d$ is the transmission to the receiving of the straight-line distance, $P_{r}$ is the received signal power, there minimum signal strength to receive information is $3.6426 \times 10^{-10}(\mathrm{w})$.

There we set transmit antenna gain is 1 , Receiving antenna gains 1 , transmitting antenna height of 1.5 , and the receiving antenna height of 1.5. Thus, by the formula (1), into the set parameters, we can get formula (2).So we can be based on the needs of the transmission range to transmit power.

$$
P_{t}=0.072 \times d^{4}, \quad(\mathrm{nW} \quad)
$$

where $d$ is the transmission distance. When the required transmission power is $P_{t}$, the consummation energy of the RREQ transmission is obtained by

$$
E_{t}=P_{t} \times T,(\mathrm{~nJ})
$$

where $T$ is the transmission time for RREQ packet.

\section{Simulation RESUltS}

In the NS-2 simulation, two parameters of the number of nodes and the transmission range are investigated for the path establishment in the networks. The network area is set by $500 \mathrm{~m} \times 500 \mathrm{~m}$. The simulation parameters are shown in Table II. By AODV routing protocols, in accordance with the different number of nodes and different transmission ranges, the successful rate of the path establishment in a network is explored.

When different transmission range is proceeded, the neighboring node in this range will received the transmission 
packet as shown in Fig. 3, where with the range of $R=250 \mathrm{~m}$ the packets can be transmitted farer than that of $R=100 \mathrm{~m}$.

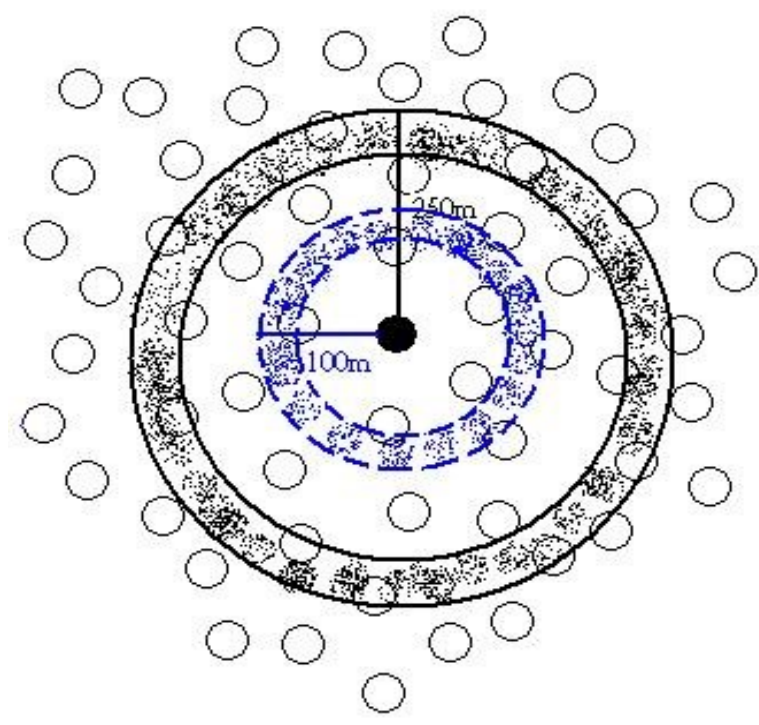

Figure 3. Broadcasting range schematic

The success rate of the path establishment is investigated for different numbers of nodes as shown in Fig. 4. From Fig. 4, we can observe that with the number of nodes $\mathrm{N}=20$, with the higher transmission range, the successful rate increases. When the number of nodes with $N=80$, the successful rate increases for transmission range $R=60$ to 100 . But it decreases for $R=120$ to 180 . When transmission range is less than 60 meters, all the success rates are equal to zero for $N \leq 100$.

The success rate for the number of nodes $10<N<100$ is shown in Fig. 5. From Fig. 5, with the transmission range $R=20$, the success rate is zero. When transmission range is greater than 80 meters, the path can be established with higher rate. But when the transmission range of 140 meters, the success rate is dropped for the number of nodes $N>70$. with the transmission range of 200 meters, the success rate begins to decline for the number of nodes $N=30$.

Therefore, from Figs. 4 and 5, it is observed that when the number of nodes is less, the transmission range should be to higher to perform more efficient connections and the success rate of the path establishment can be higher. Moreover, when the number of nodes is higher (or the nodes is denser), the transmission range should shorter to avoid collision between the RREQ packets.
TABLE II. SIMULATION PARAMETERS

\begin{tabular}{|c|c|}
\hline Network field & $500 \times 500\left(\mathrm{~m}^{2}\right)$ \\
\hline Transmission model & Non-Line-of Sight(NLOS) \\
\hline Transmission range, $R$ & $20,40,60,80,100,120,140,160$, \\
& $180,200(\mathrm{~m})$ \\
\hline Number of nodes, $N$ & $10,20,30,40,50,60,70,80,90,100$ \\
\hline Transportation protocols & TCP \\
\hline Routing protocol & AODV \\
\hline Simulation time & Random distribution \\
\hline Nodes set & 24Bytes \\
\hline RREQ packet length & 2Mbps \\
\hline Data bandwidth & \\
\hline
\end{tabular}

The comparisons of success rates are shown in Table III. From Table III, it is observed that with the number of nodes from 10 to 100, the highest success rate can be reached 82.5$95 \%$ by adequate different transmission ranges. Thus, to obtain the highest success rate for the different number of nodes, the transmission range should be selected according to the nodes density.

Moreover, the perform energy efficiency for the path establishment, the energy consumption for different transmission range $(80-200 \mathrm{~m})$ is shown in Fig. 6. From Fig. 6, it is observed that with higher number of nodes $\mathrm{N}>90$, the transmission range increases, the energy consumption increases. However, with the number of nodes $50 \leq N \leq 80$, the energy consumption is lowest for transmission range $R=100$. Moreover, Fig. 7 shows the energy consumption vs. the number of nodes $(\mathrm{N}=30-100)$. From Fig. 7, it is seen that with higher transmission range $\mathrm{R}>100$, the energy consumption increases for low number of nodes $\mathrm{N}<100$. It is due to the collision of RRQ packets. Fig. 8 shows the energy consumption vs. the number of nodes $(\mathrm{N}=60-180)$, with transmission range of 80 , energy consumption decreases for the higher number of nodes $100<N<180$.

Therefore, in the path establishment, to reduce the broadcasted RREQ packets, we can adjust the transmission range to avoid to packets collision. When the success rate is improved, the broadcast RREQ packets are reduced. Thus, the energy efficiency can be improved. 


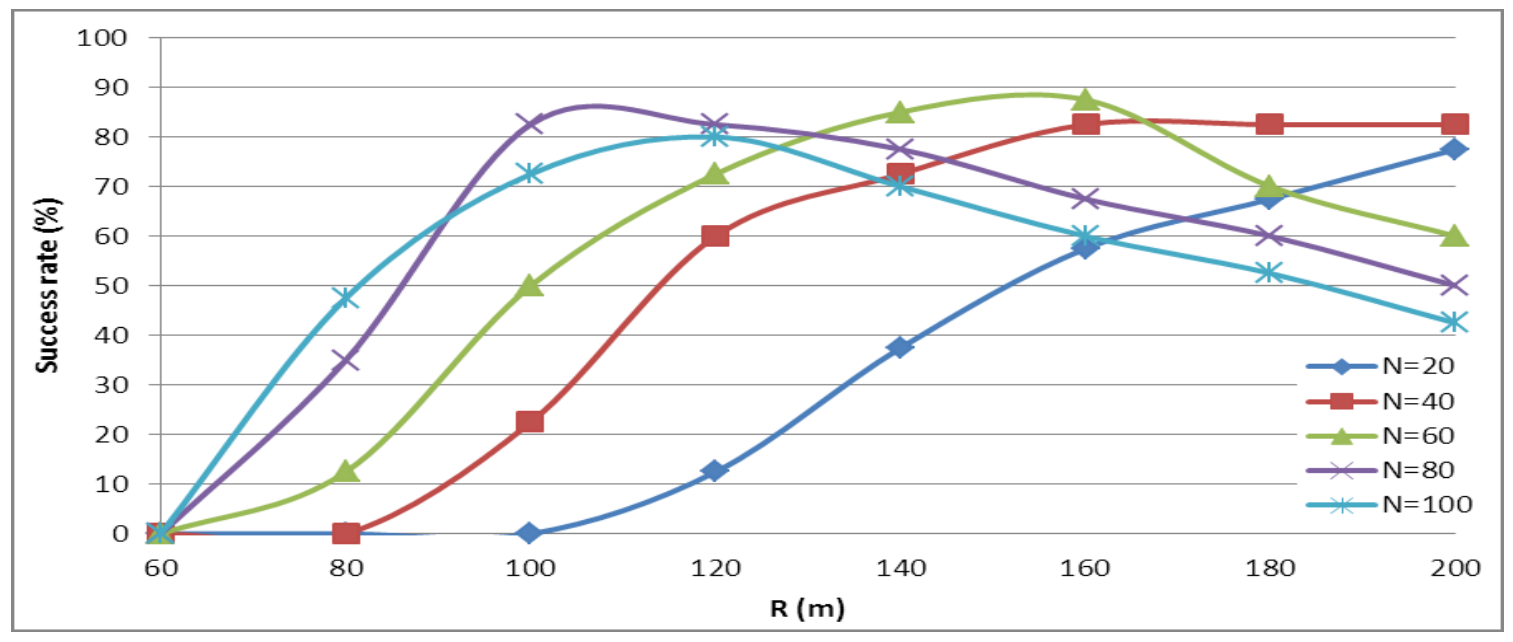

Figure 4. Path to establish the success rate of different number of nodes (20-100)

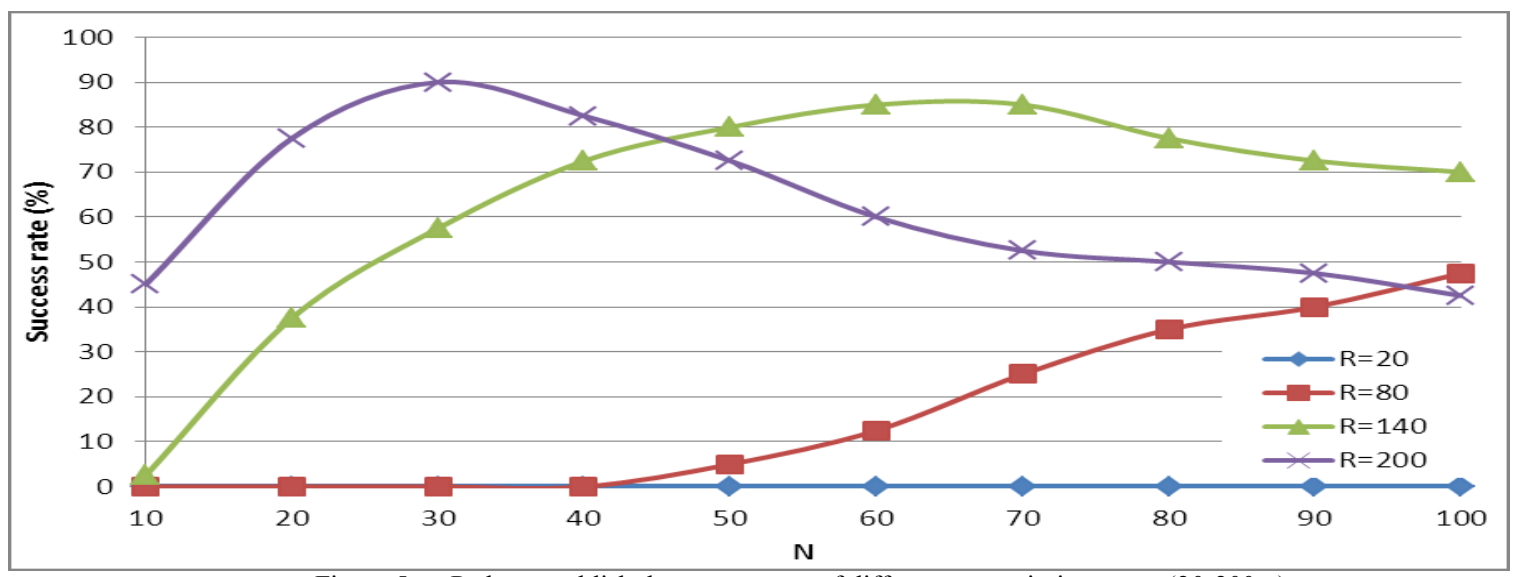

Figure 5. Path to establish the success rate of different transmission range $(20-200 \mathrm{~m})$

TABLE III. COMPARISON TABLE OF SUCCESS RATE (\%)

\begin{tabular}{|c|c|c|c|c|c|c|}
\hline $\begin{array}{l} \\
N\end{array}$ & 100 & 120 & 140 & 160 & 180 & 200 \\
\hline 10 & 0 & 0 & 2.5 & 12.5 & 25 & 45 \\
\hline 20 & 0 & 12.5 & 37.5 & 57.5 & 67.5 & 80 \\
\hline 30 & 12.5 & 37.5 & 55 & 77.5 & 95 & 90 \\
\hline 40 & 22.5 & 60 & 72.5 & 82.5 & $\overline{82.5}$ & $\overline{77.5}$ \\
\hline 50 & 40 & 67.5 & 80 & 85 & 77.5 & 72.5 \\
\hline 60 & 50 & 72.5 & $\underline{85}$ & 87.5 & 70 & 57.5 \\
\hline 70 & 70 & 80 & $\overline{85}$ & $\overline{82.5}$ & 65 & 50 \\
\hline 80 & $\underline{82.5}$ & 82.5 & 77.5 & 67.5 & 60 & 47.5 \\
\hline 90 & 77.5 & 82.5 & 72.5 & 62.5 & 55 & 47.5 \\
\hline 100 & 72.5 & 80 & 70 & 60 & 52.5 & 42.5 \\
\hline
\end{tabular}




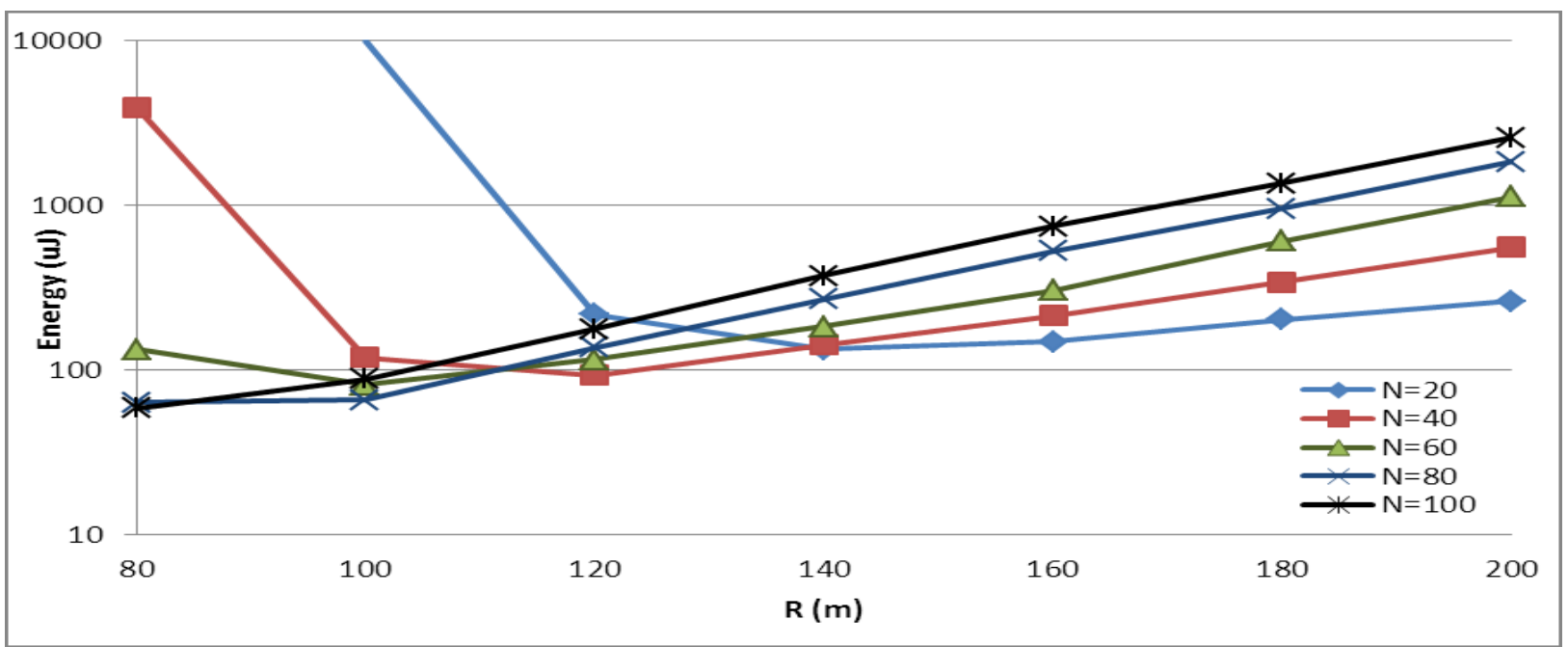

Figure 6. The comparisons of energy consumption for different transmission range (80-200m)

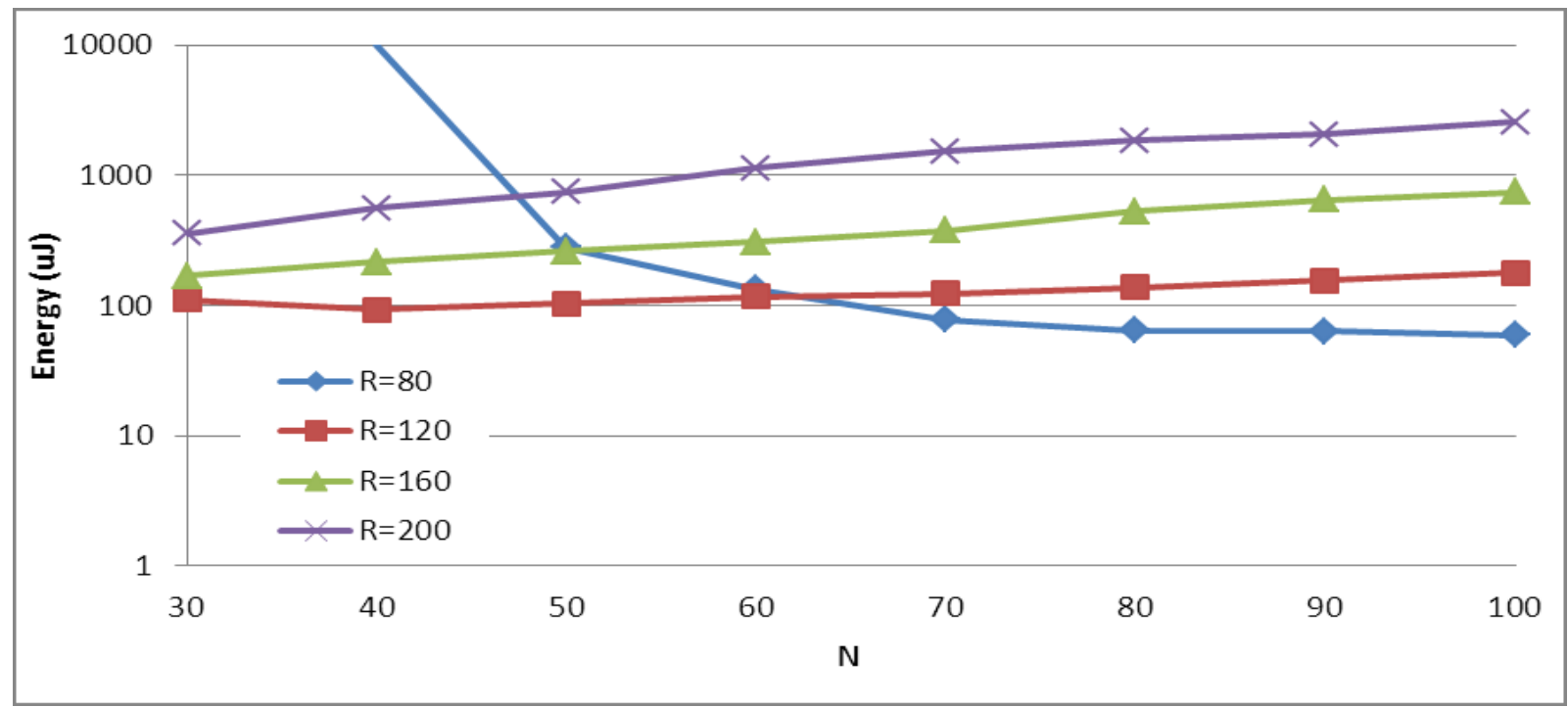

Figure 7. Energy consumption comparisons for different number of nodes (30-100) 


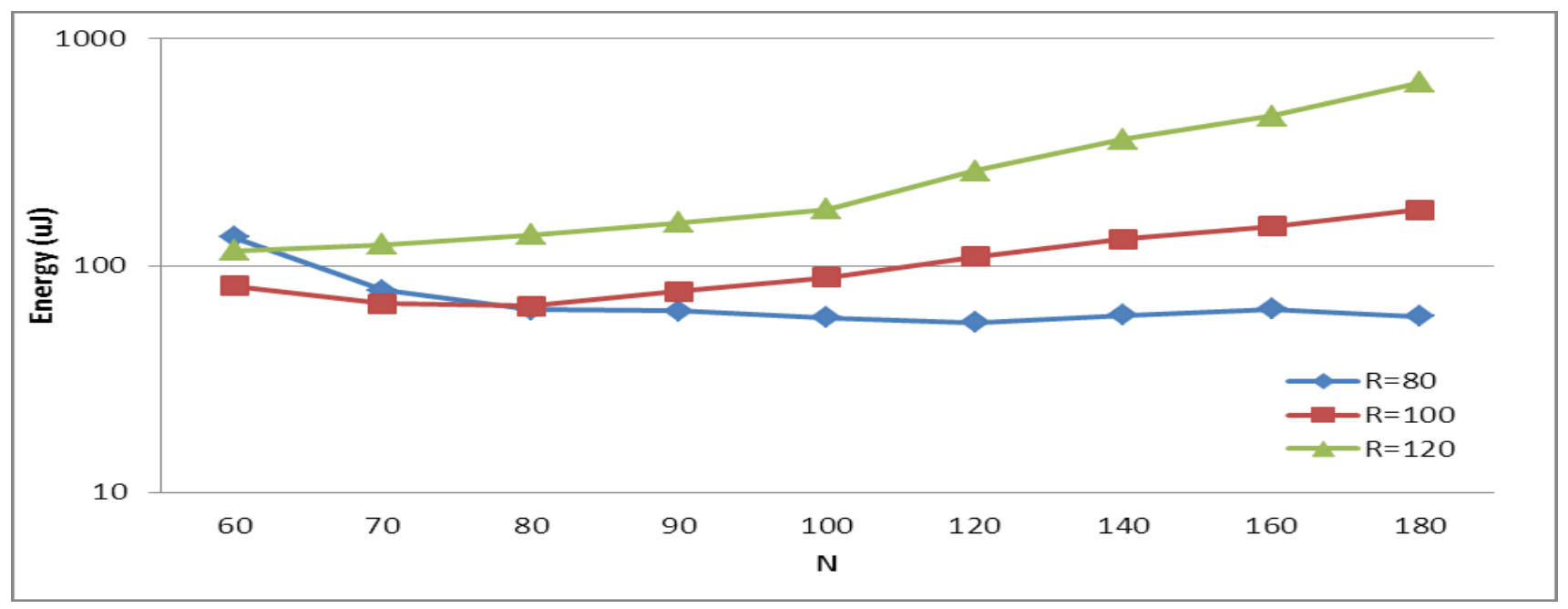

Figure 8. Energy consumption comparisons for different number of nodes (60-180)

\section{CONCLUSION}

In this paper, we investigate the path establishments of AODV protocols by computer simulations. Simulation results show that with the higher number of nodes, the transmission range for broadcasting RREQ packets should be decreased to increase the successful rate of path establishments. Moreover, when the number of nodes is 180 in the sensing field, the most energy efficiency to establish path is by using $70 \mathrm{~m}$ for transmission range to broadcast RREQ packets. In the future, we will further propose a new green energy routing algorithm to reduce the number of RREQ control packet, and reduce energy consumption, and thus enhance the performance of the network.

\section{REFERENCES}

[1] C. E. Perkins and P. Bhagwat, "Highly Dynamic Destination-Sequenced Distance-Vector Routing (DSDV) for Mobile Computers," Computer Communications Review. pp. 234-244, Oct 1994.
[2] Perkins, C.E. and Royer, E.M., "Ad Hoc On-Demand Distance Vector Routing," in Proc. of Second IEEE Workshop on Mobile Computing Systems and Applications. WMCSA '99, pp. 90-100, 1999.

[3] David B. Johnson, David A Maltz, and Yih-Chun Hu, The Dynamic Source Routing Proctocol for Mobile Ad Hoc Networks (DSR) , 19 July 2004.

[4] Rendong Bai and Mukesh Singhal, "Salvaging Route Reply for OnDemand Routing Protocols in Mobile Ad-Hoc Networks," in Proc. of MSWIM 2005, Montreal, Quebec, Canada. Oct 2005.

[5] Chonggun Kim, Elmurod Talipov, and Byoungchul Ahn, "A Reverse AODV Routing Protocol in Ad Hoc Mobile Networks," Lecture Notes in Computer Science, Vol. 4097, pp. 522-531, 2006.

[6] Shah, R.C. and Rabaey, J.M., "Energy aware routing for low energy ad hoc sensor networks," in Proc. of Wireless Communications and Networking Conference (WCNC 2002), vol. 1, pp. 350-355, 2002.

[7] I. Stojmenovic, M. Seddigh, J. Zunic, "Dominating sets and neighbor elimination-based broadcasting algorithms in wireless networks," IEEE Transactions on Parallel and Distributed Systems, pp. 14-25, 2002.

[8] J. Wu, and H. Li, "On Calculating Power-Aware Connected Dominating Sets for Efficient Routing in Ad Hoc Wireless Networks," in Proc. of the 3rd Int'1 Workshop on Discrete Algorithm and Methods for Mobile Computing and Computing and Commun., pp. 7-14, 1999.

[9] J.-H Ko, "Computer Networks Experiments - Using NS2 Simulate Multimeadia Communication and Wireless Networks", HseiGuan Publisher, 2007. (in Chinese) 